\title{
TALE-mediated epigenetic suppression of CDKN2A increases replication in human fibroblasts
}

\author{
Diana L. Bernstein, ${ }^{1}$ John E. Le Lay, ${ }^{1}$ Elena G. Ruano, ${ }^{2,3}$ and Klaus H. Kaestner ${ }^{1}$ \\ 'Department of Genetics and Institute for Diabetes, Obesity, and Metabolism, University of Pennsylvania Perelman School of Medicine, Philadelphia, Pennsylvania, USA. ${ }^{2}$ Diabetes and Obesity Laboratory, \\ August Pi i Sunyer Biomedical Research Institute (IDIBAPS), Barcelona, Spain. ${ }^{3}$ Spanish Biomedical Research Centre in Diabetes and Associated Metabolic Disorders (CIBERDEM), Barcelona, Spain.
}

\begin{abstract}
Current strategies to alter disease-associated epigenetic modifications target ubiquitously expressed epigenetic regulators. This approach does not allow specific genes to be controlled in specific cell types; therefore, tools to selectively target epigenetic modifications in the desired cell type and strategies to more efficiently correct aberrant gene expression in disease are needed. Here, we have developed a method for directing DNA methylation to specific gene loci by conjugating catalytic domains of DNA methyltransferases (DNMTs) to engineered transcription activator-like effectors (TALEs). We demonstrated that these TALE-DNMTs direct DNA methylation specifically to the targeted gene locus in human cells. Further, we determined that minimizing direct nucleotide sequence repeats within the TALE moiety permits efficient lentivirus transduction, allowing easy targeting of primary cell types. Finally, we demonstrated that directed DNA methylation with a TALE-DNMT targeting the CDKN2A locus, which encodes the cyclin-dependent kinase inhibitor p16, decreased CDKN2A expression and increased replication of primary human fibroblasts, as intended. Moreover, overexpression of p16 in these cells reversed the proliferative phenotype, demonstrating the specificity of our epigenetic targeting. Together, our results demonstrate that TALE-DNMTs can selectively target specific genes and suggest that this strategy has potential application for the development of locus-specific epigenetic therapeutics.
\end{abstract}

\section{Introduction}

Epigenetic modifications are a major determinant of gene expression programs, and inappropriate changes in these modifications can lead to a wide spectrum of diseases. Cancer is perhaps the most widely recognized disease area associated with aberrant epigenetic programs, and more recently, epigenetic changes have been implicated in neurological, metabolic, and cardiovascular diseases (1). These modifications are known to be reversible, making them attractive drug targets. To date, clinicians have relied exclusively on general inhibitors of globally expressed epigenetic regulators, which are responsible for maintaining integrity of the entire epigenome (2). Thus, unintended effects of such epigenetic inhibitors may be particularly pervasive and deleterious. Therefore, there is a need for novel tools for producing specific epigenetic changes in the laboratory to enable novel therapeutic strategies.

DNA methylation has emerged as an important mechanism governing cellular reprogramming processes such as cell differentiation, cellular senescence, and disease. In mammalian cells, DNA methylation is most abundant on cytosine residues in the context of cytosine-guanine dinucleotides, or CpGs, and, when occurring at enhancers and promoters, is frequently associated with gene repression (3). DNA methylation patterns are established by the de novo DNA methyltransferases DNMT3a and DNMT $3 \mathrm{~b}$ and propagated across cell divisions by the maintenance DNA methyltransferase DNMT1 $(4,5)$.

Conflict of interest: The authors have declared that no conflict of interest exists Submitted: May 30, 2014; Accepted: March 5, 2015.

Reference information: / Clin Invest. 2015;125(5):1998-2006. doi:10.1172/JCI77321.
In an experimental or therapeutic setting, targeted de novo DNA methylation may be accomplished by tethering of the catalytic domain of a DNA methyltransferase (DNMT) to DNA-binding proteins designed to bind specific gene loci, thereby affecting gene expression. Siddique and colleagues have pioneered this strategy by fusing DNMT catalytic subunits to an artificial zinc finger protein targeting the promoter of vascular endothelial cell growth factor A (VEGF-A) in a human cancer cell line, SOKV3 (6). However, challenges in designing artificial zinc fingers have limited the widespread use of this technology (7). Transcription activator-like effectors (TALEs) are a newer technology that is extremely modular, easy to assemble, and therefore a more efficient choice for targeted epigenome editing.

TALEs are DNA-binding proteins endogenous to bacterial plant pathogens, including the genus Xanthomonas. This class of proteins binds to regulatory regions in the host genome to modulate gene expression and promote bacteria survival. The central DNA binding domain of TALE proteins consists of a series of approximately 34 -amino acid repeats, or monomers, which are polymorphic only at positions 12 and 13 . These polymorphic residues, termed the repeat-variable-di-residue (RVD), determine DNA binding specificity, as each amino acid pair preferentially binds to 1 of the 4 nucleotides (8). Consequently, by assembly of monomers in a particular order, TALEs can be engineered to bind specific DNA sequences.

Customized TALEs have been used to modulate transcription through conjugation to activator domains, such as VP64, and repressor domains, such as the mSin interaction domain (SID) $(9,10)$. The potential for implementing TALEs to direct targeted epigenetic modifications has become increasingly recognized, as 
A

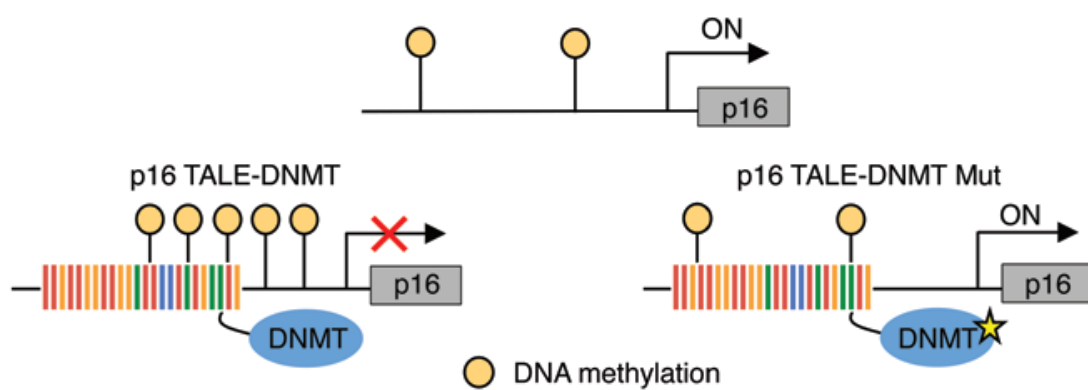

B

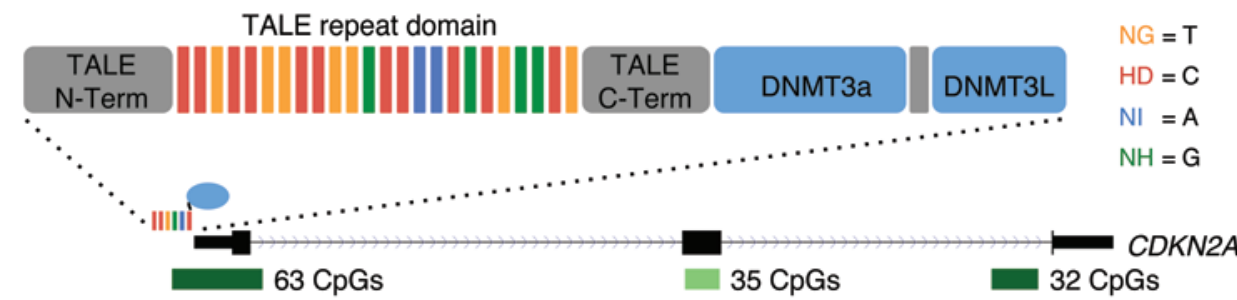

C

p16 (CDKN2A)

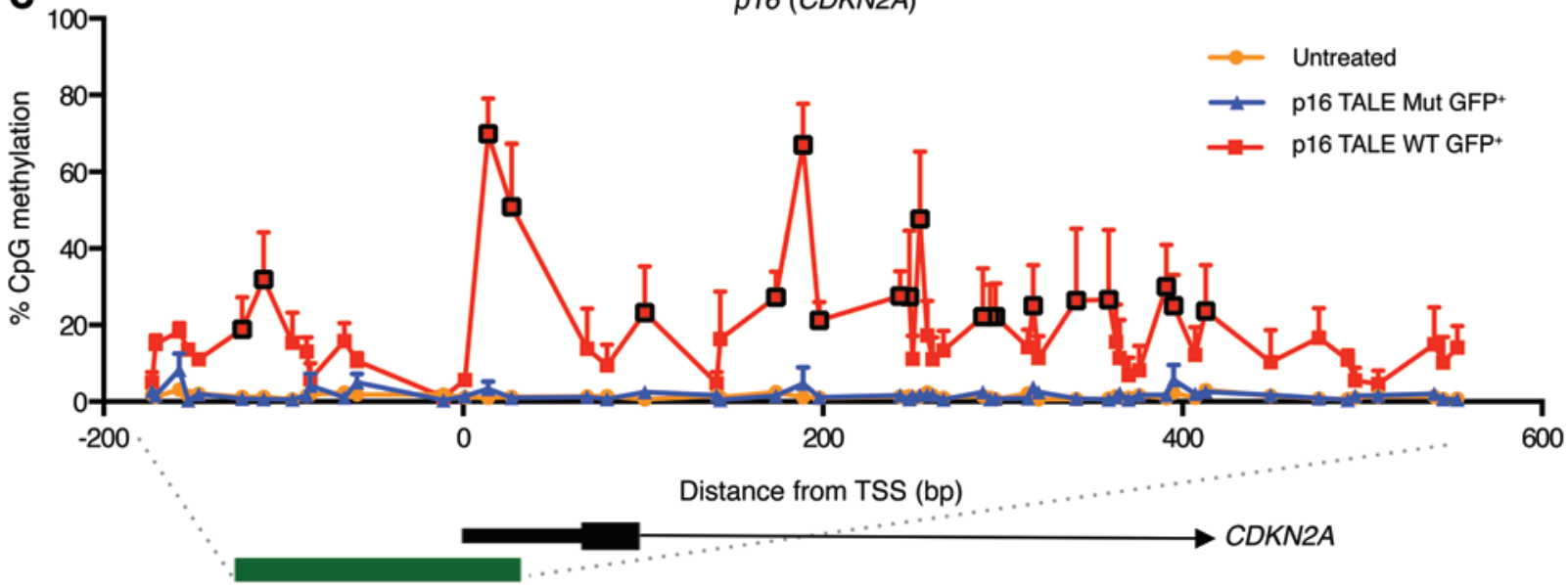

Figure 1. Targeted CpG methylation of the p16 (CDKN2A) locus using TALE-DNMT fusion proteins. (A) TALE-DNMT strategy for altering the epigenetic state of the p16 (CDKN2A) promoter. Locus-specific TALEs were fused to the catalytic domain of DNA methyltransferase (p16 TALE-DNMT), or a catalytically inactive DNA methyltransferase with the point mutation E752A (p16 TALE-DNMT Mut). (B) Detailed diagram of TALE-DNMT construct and target site in the p16 (CDKN2A) locus. Black boxes indicate the 3 exons of the p16 transcript, and green boxes indicate CpG islands. The TALE-DNMT was targeted to the $\mathrm{CpG}$ island at the promoter just before the transcription start site. The legend on the right side of the diagram indicates which nucleotide is targeted by each of the 4 different TALE repeat monomers, which are color-coded. (C) Percent methylation of individual CpGs within the CDKN2A promoter in FACS-sorted GFP-positive populations compared with untreated HeLa cells. HeLa cells were transfected with the p16 TALE-DNMT WT or p16 TALE-DNMT mutant construct and cultured for 48 hours. Cells were then FACS-sorted for GFP to isolate transfected populations. DNA methylation was quantified by sequencing of PCR-amplified bisulfite-converted genomic DNA. Graphs reflect percent DNA methylation at each CpG and its position relative to the transcription start site (TSS) (mean \pm SEM; $n=3$ ). The diagram below the graph illustrates the region of the p16 (CDKN2A) promoter that was analyzed. Data points outlined in black are significantly elevated in the p16 TALE-DNMT population compared with the p16 TALE-DNMT mutant population $(P<0.05$, multiple $t$ tests).

shown in recent studies targeting DNA and histone demethylation $(11,12)$. However, this approach has not yet been used to repress gene expression by targeted DNA methylation. A key hurdle in widespread use of TALEs is that they are incompatible with lentivirus technology, a common approach in stable transmission of genes into host genomes, particularly with primary cells. The highly repetitive sequences of the TALE modules have a strong tendency to recombine (13), and we addressed this issue by reengineering the TALE repeat moieties to minimize direct DNA repeats without altering the coding sequence.

Our primary target for development of this novel "TALEDNMT" strategy was the cell cycle inhibitor p16, which is encoded by the CDKN2A gene (Figure 1A). p16 is a universal regulator of cellular senescence, and CDKN2A was found to be the most common locus associated with age-related disease in a metaanalysis of GWAS conducted by Jeck and colleagues $(14,15)$. Prior studies have shown that CDKN2A is regulated by DNA methylation, and decreasing p16 levels might aid in coaxing terminally differentiated cells back into the cell cycle, allowing for cell expansion for experimental or cell therapeutic uses $(16,17)$.

Thus, we sought to target DNA methylation to the p16 (CDKN2A) locus using lentiviral delivery of TALE-DNMT fusion proteins to repress gene expression and thereby increase cellular proliferation. We also characterized the specificity of TALE- 
A

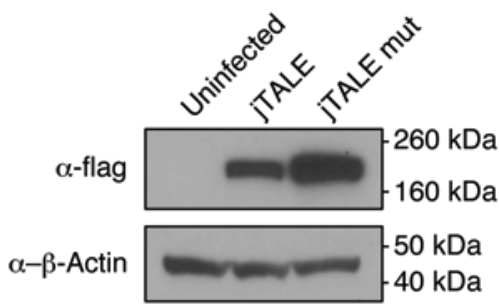

B

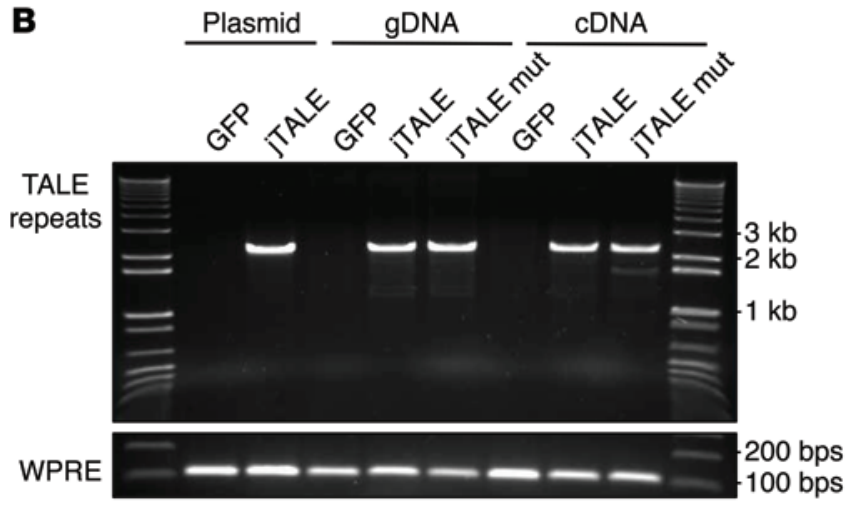

C

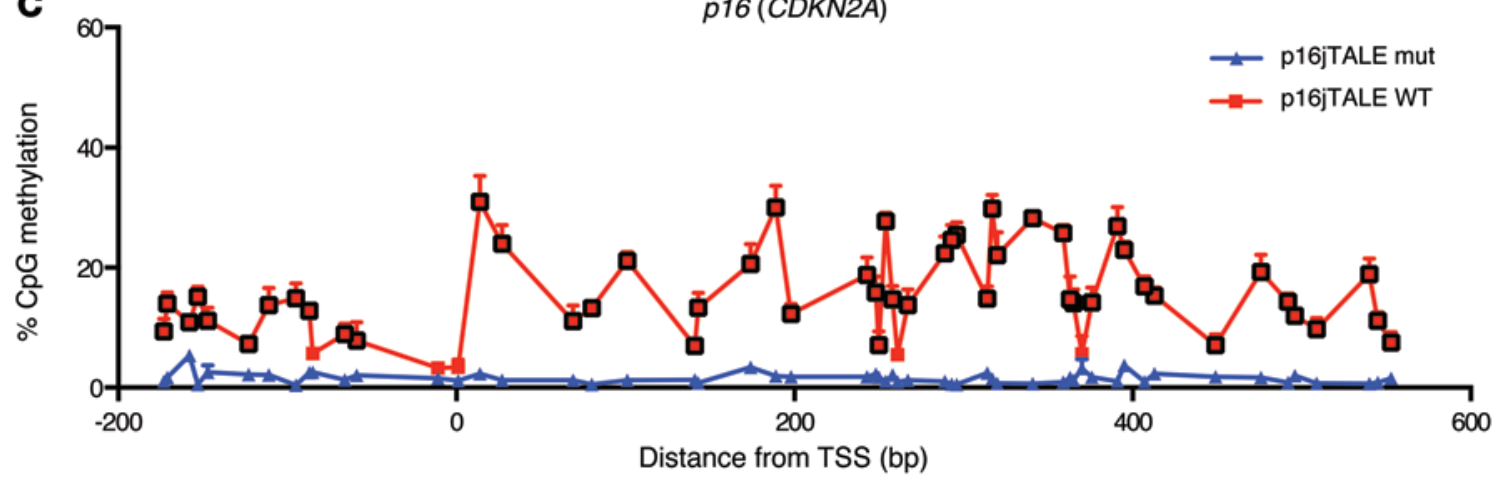

Figure 2. Minimizing direct repeats permits lentiviral expression of TALE fusion proteins. HeLa cells were infected with p16 jumbled TALE-DNMT, p16 jumbled TALE-DNMT mutant, or GFP control lentiviruses and harvested after 4 days. (A) Western blot of HeLa cells infected with p16 jTALE-DNMT or p16 jTALE-DNMT mutant lentivirus showing production of the full-length protein. (B) PCR amplification of the full-length TALE repeat moiety from genomic DNA (gDNA), demonstrating integration of the intact construct into the host genome, and from cDNA, demonstrating transcription of full-length mRNA, in infected HeLa cells. Amplification of plasmid DNA is shown as a reference. jTALE, jumbled TALE; WPRE, woodchuck hepatitis virus post-transcriptional regulatory element. (C) Percent DNA methylation of the p16 (CDKN2A) locus in HeLa cells infected with p16 jTALE-DNMT WT and p16 jTALE-DNMT mutant lentivirus (mean $\pm \mathrm{SEM} ; n=3$ ). Data points outlined in black indicate $\mathrm{CpCs}$ in which DNA methylation is significantly elevated in p16 jTALE-DNMT WTinfected cells compared with p16 jTALE-DNMT mutant-infected cells $(P<0.05$, multiple $t$ tests).

mediated epigenetic modifications, which, to our knowledge, has not been reported to date. Here, we show that epigenetic targeting of a single locus can indeed alter its gene expression and cellular functions without appreciable "off-target" effects, thus demonstrating that the phenotype of primary human cells can be altered using epigenetic tools.

\section{Results}

Custom TALE-DNMT fusion proteins direct DNA methylation to target loci. Sanjana and colleagues have described a protocol for assembly of custom TALEs using monomer templates and TALE cloning backbones (18). We modified these TALE constructs by conjugating a DNA methyltransferase catalytic subunit consisting of the C-termini of DNMT3a and DNMT3L (6) to the C-terminus of the TALE protein. The cloning backbone contains EGFP and was further modified to include a 3x FLAG-tag, modifications that we used for cell sorting and protein detection, respectively. We also constructed a catalytically inactive TALE-DNMT as a negative control by introducing a point mutation in the DNMT3a subunit at the E752A position (19). TALE monomers were assembled into the backbone-cloning vector through a series of Golden Gate digestion-ligation reactions (18). Following this protocol, we constructed TALE-DNMTs targeting 24-bp sequences within the CDKN2A promoter (20). The TALE-DNMT illustrated in Figure 1B is engineered to bind the target sequence $5^{\prime}$-ССТССТТССТTGCCAACGCTGGCT-3', at position -28 to -4 upstream of the p16 (CDKN2A) transcription start site. The complete coding sequence of the p16 TALE-DNMT is provided in Supplemental Figure 1 (supplemental material available online with this article; doi:10.1172/JCI77321DS1).

To test our strategy, we transfected HeLa cells, a human cervical adenocarcinoma cell line, with p16 TALE-DNMT WT and mutant expression constructs and compared DNA methylation of the $C D K N 2 A$ locus between the 2 transfected populations, and also untreated HeLa cells. Cells were collected 48 hours after transfection, and FACS-sorted for GFP to isolate transfected populations. Average transfection efficiency was $12.1 \%$ and $14.6 \%$ for the WT and mutant constructs, respectively. DNA methylation was then evaluated using bisulfite conversion of genomic DNA followed by PCR amplification and high-throughput sequencing. Strikingly, we found that transfection of a single TALE-DNMT construct is sufficient to dramatically alter DNA methylation across the entire $\mathrm{CpG}$ island within the CDKN2A promoter (Figure 1C). DNA methylation was significantly elevated in the p16 TALE-DNMT GFP-positive cells compared with both mutant GFP-positive and untreated cells $(P<0.0001)$. On average, DNA methylation increased by $17 \%$ across the entire $\mathrm{CpG}$ island, and by as much as $66.5 \%$ at individual CpGs, when WT- and mutant- 

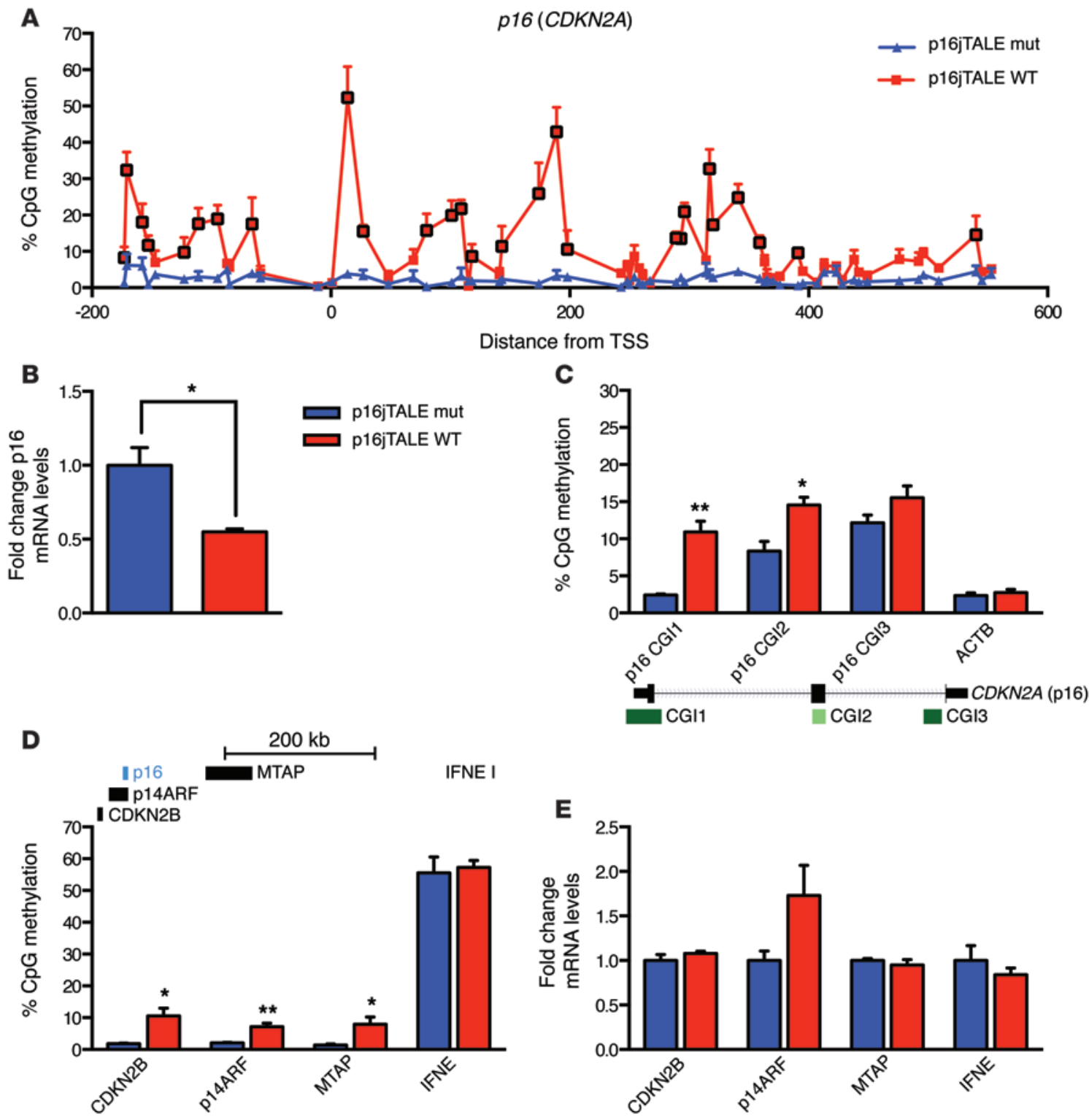

Figure 3. Targeted CpG methylation at the p16 (CDKN2A) locus results in decreased gene expression in primary human cells. Primary human fibroblasts were transduced with p16 jTALE-DNMT WT or p16 jTALE-DNMT mutant lentiviruses and incubated for 4 days. (A) Percent DNA methylation of CpGs within the $p 16$ (CDKN2A) promoter region. Graphs reflect percent DNA methylation at each CpC (mean $\pm \mathrm{SEM} ; n=3$ ) and position relative to the transcription start site. Data points outlined in black are significantly elevated in the p16 jTALE-DNMT population compared with the p16 jTALE-DNMT mutant population $(P<0.05$, multiple $t$ tests). (B) p16 transcript expression in fibroblasts treated with p16 jTALE-DNMT WT or mutant lentiviruses relative to the mutant negative control. Expression levels were normalized to HPRT1 mRNA levels (mean $\pm \mathrm{SEM} ; n=3$ ). ${ }^{*} P<0.05$, 2-tailed $t$ test. (C) Average percent DNA methylation of CpGs at each CpG island within the p16 (CDKN2A) locus and at $\beta$-actin (ACTB), a housekeeping gene located on a different chromosome. The diagram below the graph illustrates the position of CpG islands at the p16 (CDKN2A) locus (mean $\pm \mathrm{SEM} ; n=3$ ). ${ }^{*} P<0.05,{ }^{* *} P<0.01,2$-tailed $t$ test. (D) Average percent methylation at genes adjacent to $p 16$ (CDKN2A), as described in $\mathbf{C}$. The diagram above the graph indicates the position of each gene relative to $p 16$ (CDKN2A). (E) mRNA expression of genes adjacent to p16 (CDKN2A) in lentivirally transduced human fibroblasts, determined as described in B.

transfected populations were compared (Figure 1C). There was no difference between p16 TALE-DNMT mutant-transfected and untreated populations, confirming that the E752A mutation completely eliminates the catalytic activity of the enzyme.

Despite the dramatic increase in DNA methylation at the CDKN2A promoter, p16 expression decreased by only a small amount in p16 TALE-DNMT WT-transfected HeLa cells compared with mutant and uninfected populations (data not shown). This is likely due to the fact that p16 expression is often upregulat- ed in cervical cancers, and may be subject to aberrant regulatory mechanisms in these transformed cells $(21,22)$. Thus, we hypothesized that a primary human cell line might be a more suitable system to study functionality at this particular target.

Minimizing direct repeats in TALE modules permits lentiviral delivery. We next considered alternate strategies for gene delivery that might be more suitable to targeting primary human cells, which are often difficult to transfect even ex vivo. Lentiviral vectors provide an efficient method for stably introducing genes into 
A

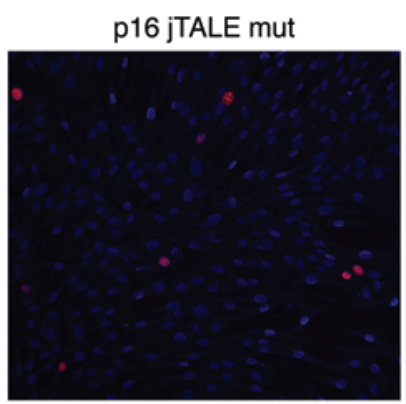

p16 jTALE WT

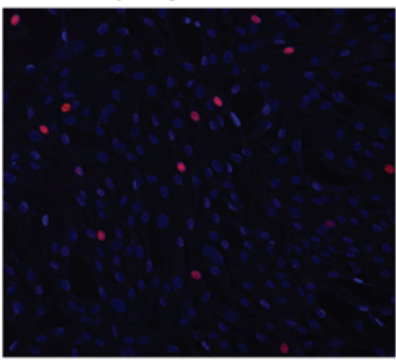

B

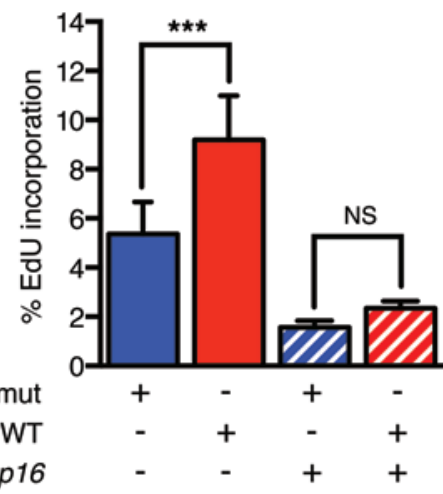

C

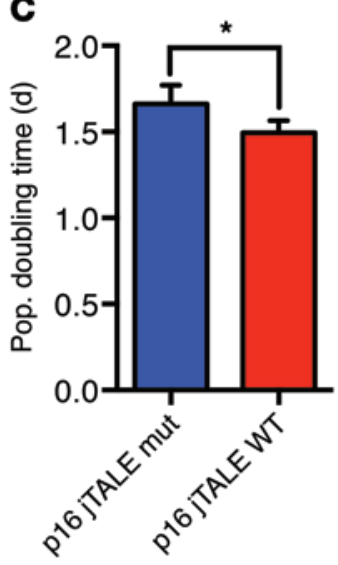

D

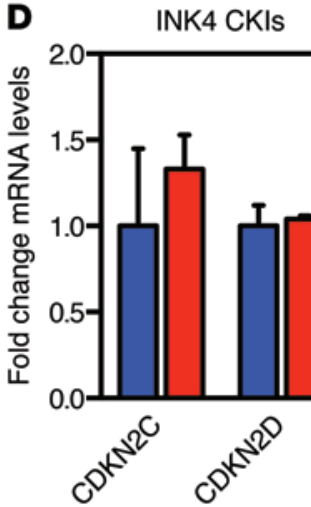

E

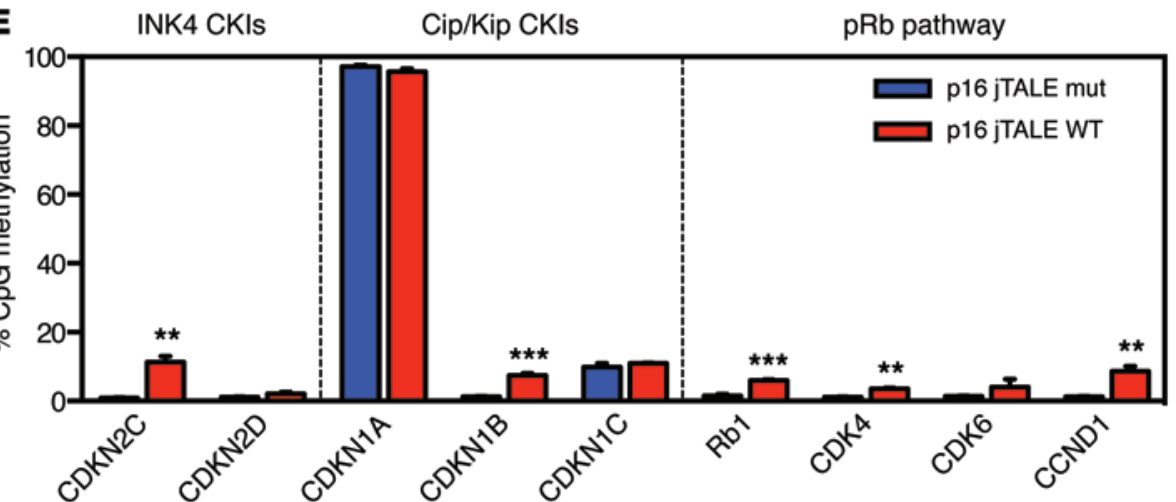

Figure 4. Alterations in p16 levels due to p16 TALE-DNMT result in increased proliferation in primary human cells. (A) Representative immunofluorescence image of EdU incorporation in human fibroblasts infected with WT or mutant p16 jTALE-DNMT lentivirus. After 72 hours of infection, cells were incubated with EdU for 1 hour and stained for EdU. Cell nuclei are stained blue (DAPI), and EdU-positive nuclei are stained red. Images were taken at $\times 10$ magnification $(n=7)$. (B) Percent EdU incorporation of cells infected with p16 jTALE-DNMT WT or mutant lentivirus, with $(n=4)$ or without $(n=7)$ coinfection of CMV-p16 lentivirus. Three random images were counted for each biological replicate (mean \pm SEM). *** $P<0.001$; NS, not significant; 2 -tailed $t$ test. (C) Population doubling time of human fibroblasts infected with p16 jTALE-DNMT WT or mutant lentivirus (mean \pm SEM; $n=4$ ). ${ }^{*} P<0.05,2$-tailed $t$ test. (D) Transcript levels of cell cycle regulators in human fibroblasts transduced with WT p16 jTALE-DNMT lentivirus relative to mutant lentivirus. Expression was normalized to HPRT1 mRNA levels (mean \pm SEM; $n=3$ ). Two-tailed $t$ test. (E) Average percent DNA methylation of CpGs at the nearest CpG island of cell cycle regulators evaluated in D. Average DNA methylation was measured by PCR amplification of bisulfite-converted genomic DNA followed by highthroughput sequencing (mean $\pm \mathrm{SEM} ; n=3$ ). ${ }^{* *} P<0.01,{ }^{* *} P<0.001$, 2-tailed $t$ test. CKI, cyclin-dependent kinase inhibitor.

host genomes of multiple cell types. However, standard TALE technology is incompatible with lentiviral delivery because of the large number of tandem repeats in the TALE moiety that lead to sequence loss by DNA recombination (13). In order to promote efficient integration of intact TALEs into host genomes using lentivirus, we used the degeneracy of the genetic code to minimize direct repeats across the TALE DNA binding domain to build “jumbled” TALE-DNMTs (jTALE-DNMTs) (Supplemental Figure 2), similar to the strategy pursued by Yang and colleagues (23).
Furthermore, in order to accommodate the size limitation of the lentiviral genome, we made several modifications to the TALEDNMT constructs by decreasing the number of repeats from 24 to 18 , such that the revised TALE targets the sequence $5^{\prime}$-TCCTTGCCAACGCTGGCT-3'. We also truncated the promoter and removed the EGFP sequence.

We prepared lentivirus for WT and mutant p16 jTALE-DNMT constructs and tested their functionality in HeLa cells. Western blots confirmed that full-length jTALE-DNMT protein was indeed 
produced following infection (Figure 2A). In order to demonstrate that the entire, unrecombined jumbled TALE repeat moiety was integrated into the host genome, we PCR-amplified the repeat region from genomic DNA of transduced cells. We also amplified the jTALE repeat region from cDNA to determine that the fulllength repeat region was transcribed (Figure 2B). Together, these assays confirmed integration and expression of full-length TALE constructs. We then established that p16 jTALE-DNMT lentiviruses target $\mathrm{CpG}$ methylation at the CDKN2A locus, with an average increase of $13.8 \%$ in the p16 jTALE-DNMT-infected population compared with the mutant (Figure 2C). The lesser extent of DNA methylation in the lentivirus infections compared with transfected cells is likely due to the fact that cells could not be sorted into infected populations. These data demonstrate convincingly that jumbled TALE repeats can be administered stably using lentiviral vectors, removing a significant obstacle for widespread application of the TALE technology.

Targeted DNA methylation in primary human cells results in decreased gene expression of the target gene. Having successfully developed jTALE-DNMTs for lentiviral delivery, we next tested whether TALE-DNMTs can methylate the p16 (CDKN2A) locus in primary human fibroblasts. After infection with WT and mutant p16 jTALE-DNMT constructs, DNA methylation was again evaluated by sodium bisulfite conversion followed by PCR amplification and high-throughput sequencing. Average DNA methylation was significantly increased by approximately $10 \%$ across the CDKN2A $\mathrm{CpG}$ island in fibroblasts infected with WT versus mutant p16 jTALE-DNMTs $(P<0.005)$, with several CpGs showing increases in methylation of $30 \%-50 \%$ (Figure $3 \mathrm{~A}$ ). To evaluate the functional consequence of increased $\mathrm{CpG}$ methylation at this locus, we measured p16 mRNA levels and found an approximately $50 \%$ decrease in $p 16$ expression in p16 jTALE-DNMT-infected fibroblasts relative to mutant-infected fibroblasts $(P<0.05)$ (Figure $3 \mathrm{~B})$. In order to validate the broad applicability of the TALE-DNMT strategy, we also tested the p16 TALE-DNMT lentiviruses in primary human coronary artery smooth muscle cells, and showed that p16 jTALE-DNMT WT-infected cells had increased DNA methylation and a corresponding decrease in p16 (CDKN2A) expression compared with mutant-infected cells (Supplemental Figure 3). As further validation of our strategy, we also designed a new p16 TALE-DNMT targeted to the p16 (CDKN2A) promoter 118-136 bp upstream from the transcription start site (5'-TAACAGAGTGAACGCACT-3'). This additional TALE-DNMT, p16 TALE-DNMT.2, also increased DNA methylation and led to even stronger repression of $p 16$ transcription when WT- and mutantinfected cells were compared (Supplemental Figure 4). Remarkably, this stronger gene repression was associated with fewer affected CpGs, suggesting that specific CpGs are more relevant than others. In sum, the overall TALE-DNMT strategy can be extended to multiple primary human cell types and multiple target DNA binding sites.

To further characterize the effects of the p16 TALE-DNMT locally and genome-wide, we measured DNA methylation at the 2 other CpG islands within the $p 16$ (CDKN2A) locus, and at $\beta$-actin, a housekeeping gene located on a different chromosome (Figure 3C). Interestingly, we found that the effect of the TALE-DNMT on average DNA methylation decreased with distance from the tran- scription start site at the $p 16(C D K N 2 A)$ locus, as DNA methylation increased significantly by $6 \%$ at CGI2, but not significantly at CGI3. The $\beta$-actin locus on chromosome 7 was completely unaffected.

Given the increase in DNA methylation observed at the adjacent $\mathrm{CpG}$ island, we considered the possibility that other nearby genes might display altered levels of DNA methylation (Figure $3 D)$. Indeed, we measured increased methylation in several genes adjacent to p16 (CDKN2A), including p14ARF, another transcript within the $C D K N 2 A$ locus, $C D K N 2 B$, and MTAP. No changes were observed in IFNE. We next measured mRNA levels at these genes to determine the functional consequence of the small increase in DNA methylation and observed no changes in the mRNA levels of CDKN2B, or MTAP and IFNE (Figure 3E). We also analyzed expression of the 3 other transcripts at the CDKN2A locus and did not observe a significant change in expression of $p 14 A R F$ (Figure $3 \mathrm{E}$ ), while $p 12$ and $p 16 \gamma$ were not detectable by quantitative PCR in human fibroblasts.

DNA methylation of the p16 (CDKN2A) locus results in increased replication in primary human cells. Our goal in decreasing p16 expression was to more readily permit entry into the cell cycle. Therefore, we next evaluated rates of DNA replication in primary human fibroblasts infected with WT and mutant p16 jTALEDNMTs. Following infection and subsequent incubation for 72 hours, we measured incorporation of the thymidine analog 5-ethynyl-2'-deoxyuridine (EdU) by immunofluorescence staining after a 60-minute incubation period. p16 jTALE-DNMTs increased DNA replication nearly twofold in the WT-infected compared with mutant-infected populations (Figure 4, A and B). In order to confirm that the altered proliferation observed in $\mathrm{p} 16$ jTALE-DNMT-transduced human fibroblasts was specifically due to suppression of p16 transcription, and not some unknown offtarget effect, we restored p16 levels by coinfection with a lentivirus driving $p 16$ expression under control of the CMV promoter. As shown in Figure 4B, re-expression of p16 completely ablated the pro-proliferative effect of p16 jTALE-DNMT activity, providing strong evidence that increased replication in human fibroblasts was indeed caused by reduced p16 levels. Since p16 mediates progression through the G1 phase of the cell cycle, we also wanted to confirm that increased DNA replication rates translated into cellular proliferation. We therefore calculated population doubling time of infected human fibroblasts and found that this was reduced by $10 \%$ in the p16 jTALE-DNMT WT-infected cells compared with mutant (Figure 4C).

To further eliminate the possibility that increased proliferation of epigenetically targeted human fibroblasts might be impacted by other cell cycle regulators, we determined the expression levels of a panel of cyclin-dependent kinase inhibitors, cyclin-dependent kinases, and other molecules related to the p16 inhibitory pathway. As shown in Figure 4D, none of these cell cycle regulators were affected by p16 jTALE-DNMT infection comparing WT- and mutant-infected populations, attesting to the specificity of our strategy. Although we demonstrated that p16 TALE-DNMT infection does not affect expression of other cell cycle regulators, we also assessed DNA methylation at these loci. We found that some genes showed increased methylation while others did not (Figure $4 \mathrm{E}$ ). Since changes across cell cycle regulators are inconsistent, we cannot say for certain whether or not these changes are a direct 
consequence of p16 TALE-DNMT activity, or an indirect consequence of decreased p16 expression and accelerated proliferation. In summary, targeting DNA methylation to the p16 (CDKN2A) promoter led to changes in expression exclusively of the $p 16$ transcript, resulting in increased cell proliferation, without altering the activity of other cell cycle regulator genes.

\section{Discussion}

We have demonstrated that customized TALEs can be used to direct DNA methylation to specific gene loci, and thereby decrease gene expression. We selected p16 as our primary target because of its role in mediating cellular senescence with the idea that epigenetic suppression of p16 might facilitate cell cycle entry in terminally differentiated cells in the context of regenerative medicine. In developing this novel technology, we used a primary human fibroblast cell line that is not transformed, in contrast to the majority of human cells lines, which are often subject to dysfunctional cell cycle regulation. Importantly, even in this readily dividing cell population we have shown that increasing DNA methylation at a single cell cycle inhibitor gene locus is sufficient to increase cellular proliferation. Further, the observed changes in replication rate were entirely dependent on suppression of the intended gene product, p16. We have also proven that these targeted epigenetic modifiers can be delivered using lentiviral vectors, which will dramatically expand the breadth of their application in different biological systems. We validated our findings of TALE-DNMT-mediated targeted DNA methylation and p16 suppression in primary human coronary artery smooth muscle cells, and with an independent TALE-DNMT construct targeting the p16 (CDKN2A) locus, attesting to the broad applicability of this technology. We have shown that the TALE-DNMT strategy is a powerful and robust tool that can be used to change cell fate.

We also found that nearby loci are more likely to be susceptible to off-target effects by TALE-DNMTs than distal loci, which until now has been an underreported consequence of TALE-mediated epigenetic modifiers. These "near-target" effects appear to be distance dependent, and in our case functionally irrelevant as altered gene expression was detected only at the target gene, CDKN2A. It is likely that the "near-target" effects can be attributed to the 3-dimensional structure of chromatin. Chromatin interactions such as chromatin looping may bring nearby loci into the vicinity of the TALE-DNMT construct, permitting DNA methylation in the absence of direct TALE DNA binding. We also observed small increases in DNA methylation at some but not all cell cycle inhibitors and other cell cycle regulators implicated in the p16/ pRb pathway. Critically, these changes did not impact gene activity. Nevertheless, future efforts will be directed toward further improving specificity by strategies such as attenuating DNMT subunit catalytic activity, or splitting the DNMT domain, similar to the TALEN system, such that 2 separate TALE fusion proteins are required for methyltransferase activity. Also, the TALE-DNMT strategy may be further optimized through large-scale screens of TALEs to determine whether certain target binding sites are more effective than others.

While there are several techniques being developed for directing epigenetic modifiers to specific genomic loci, they differ in their DNA targeting strategies. Current approaches include zinc fingers, TALEs, and clustered regularly interspaced short palindromic repeat (CRISPR)/CRISPR-associated protein 9 (Cas9). Zinc fingers represent one of the earliest examples of engineered DNA-binding proteins, and have been coupled to a wide range of effector domains, including DNA methyltransferases, as discussed earlier. Zinc finger DNA binding modules interact with a series of 3-bp elements, somewhat limiting potential target sequences. Therefore, TALEs have largely replaced zinc fingers in these efforts, since they are modular, inexpensive, and quick to make. The CRISPR/Cas9 system has emerged as an exciting new tool for genome editing, as the Cas9 nuclease can be directed to target DNA sequences by 20-bp small guide RNAs, eliminating the need to engineer sequence-specific DNA-binding proteins. While most work to date has focused on genomic engineering, the field is rapidly evolving into the gene expression space, using a catalytically inactive or "dead" variant of the Cas9 nuclease, dCas9. In fact, the concept of using Cas9 toward targeted epigenetic modifications has already been proposed, although not yet demonstrated (24). Despite differences in underlying mechanisms, any of these systems are subject to potential off-target effects, and improving specificity is an ongoing challenge in the field. The preferred method is likely to be determined in a context-dependent manner.

Here, we demonstrate the utility of TALE-directed DNA methylation as a strategy for altering the epigenetic state in a targeted, locus-specific fashion. We have amended this strategy to accommodate lentiviral delivery to primary human cells, and have shown that this system can be used to alter cellular behavior. We also shed light on the specificity of TALE-mediated epigenetic targeting, which is an ongoing area of future investigation. This study may have widespread implications for investigating gene regulation, and in developing novel therapeutic strategies for correcting aberrant gene expression in disease.

\section{Methods}

TALE target selection and construction. Twenty-four-base pair TALE target sequences within the CDKN2A promoter were selected using the Web-based tool TAL Effector-Nucleotide Targeter (TALE-NT) 2.0, which optimizes unique binding sites within a specified target region $(8,25)$. The sequence targeted in the present work was 5'-CСTCCTTCCTTGCCAACGCTGGCT-3'. Cloning vectors and TALE repeat monomer plasmids were obtained from the TALE Toolbox kit (Addgene). pTALETF vectors were modified to replace the VP64 domain with FLAG-tagged DNMT3a-3L by cloning. Following target selection, the 24 corresponding monomers were assembled into a modified TALE backbone containing either the DNMT3a-3L catalytic subunit or the mutant DNMT3a-3L catalytic subunit through a series of Golden Gate digestion-ligation reactions, as described by Sanjana and colleagues (18). The mutant DNMT catalytic domain contained a catalytically inactive DNMT3a subunit due to a point mutation, E752A (19). The DNMT3a-3L DNA sequence was synthesized by Eurofins MWG Operon and cloned into the TALE backbone by restriction enzyme digestion followed by ligation. PCR primers including the E752A point mutation were used to amplify a DNA fragment from the TALE-DNMT plasmid containing the point mutation, and the fragment was subsequently cloned into the TALE-DNMT backbone by restriction enzyme digestion followed by ligation to generate the 
mutant construct. The point mutation is underlined in the reverse primer (PCR primers: forward, 5'-CAAGCCCCAAGAAGAAGAGA-3'; reverse: 5'-CCCATGGCCACCACATTGGCAAAGAG-3').

Jumbled TALE lentivirus design and construction. Jumbled TALEs were designed by disruption of direct repeats greater than 11 nucleotides using the degeneracy of the genetic code to change DNA sequence without altering protein coding sequence. In order to accommodate the size limitation of the lentiviral genome, the TALE target sequence was decreased to 18 bases instead of 24, a minimal CMV promoter was used, and EGFP was removed. The 18-bp sequence targeted with the jumbled TALE was 5'-TCCTTGCCAACGCTGGCT-3'. Series of plasmids containing jumbled sequences for 6 monomers were ordered from Eurofins MWG Operon with appropriate restriction sites such that these hexamers could be incorporated into the protocol described by Sanjana and colleagues at a Golden Gate digestion-ligation step. TALE-DNMT constructs were then cloned into a lentiviral vector obtained from The Wistar Institute Protein Expression Core (Philadelphia, Pennsylvania, USA). Lentiviruses were also prepared by The Wistar Institute Protein Expression Core.

Transfection of HeLa cells. HeLa cells (ATCC) were seeded at a density of $5 \times 10^{6}$ cells per $10 \mathrm{~cm}^{3}$. After 24 hours, cells were transfected with $12.5 \mu \mathrm{g}$ of plasmid DNA using Lipofectamine 2000 reagent (Life Technologies) according to the manufacturer's protocol. Forty-eight hours after transfection, cells were trypsinized with $0.25 \%$ trypsin plus EDTA and dispersed for FACS sorting for GFP. FACS was performed by the Flow Cytometry and Cell Sorting Resource Laboratory at the University of Pennsylvania. Sorted populations were harvested using the Qiagen AllPrep DNA/RNA Mini Kit.

DNA methylation analysis. Genomic DNA was bisulfite-converted using the Qiagen EpiTect Bisulfite Kit (Qiagen $\mathrm{GmbH}$ ), and target loci were PCR-amplified using the PyroMark PCR Kit (Qiagen GmbH). Four primer pairs were designed to PCR-amplify across the entire CDKN2A $\mathrm{CpG}$ island within the promoter region. Additional primer pairs were also designed to amplify regions within each control locus. Primers were designed to amplify approximately 250- to 300-bp regions at the $\mathrm{CpG}$ island closest to the transcription start site of each gene. In instances when no $\mathrm{CpG}$ island was present, a sequence within the gene promoter was chosen. All primer sequences and genomic coordinates for each amplicon are listed in Supplemental Table 1. PyroMark PCR reactions were carried out according to the manufacturer's instructions. DNA sequencing libraries were prepared with the automated Ovation SP+ Ultralow DR Multiplex System (NuGEN Technologies Inc.) and subsequently sequenced on an Illumina MiSeq with 150-bp paired-end reads. DNA sequences were aligned to an in silico bisulfiteconverted human genome using the BS Seeker program and analyzed by the Next Generation Sequencing Core at the University of Pennsylvania (26). Only CpGs with sequence coverage greater than 1,000 reads were considered. Average DNA methylation across regions was analyzed by 1-way ANOVA with Tukey's correction for multiple comparisons when comparing more than 2 groups, and by a 2-tailed $t$ test when comparing 2 groups. Individual CpGs were compared by multiple $t$ tests $(P<0.05)$. All next-generation sequencing data were deposited to the Gene Expression Omnibus (GEO) public database according to MINSEQE standards (accession no. GSE66456).

Quantitative real-time PCR for gene expression. To assess mRNA levels, RNA extracted with the Qiagen AllPrep DNA/RNA Mini Kit was reverse-transcribed using SuperScript II Reverse Transcriptase
(Life Technologies) to synthesize cDNA. Quantitative RT-PCR was performed on the Agilent Technologies Stratagene Mx3000P using 2x Brilliant III SYBR Green qPCR Master Mix plus ROX reference dye (Agilent Technologies). Thermal profiles were set according to the manufacturer's protocol. mRNA levels were normalized to HPRT1. Quantitative PCR primer sequences are listed in Supplemental Table 2. Differences in mRNA levels were compared by 2-tailed $t$ tests $(P<0.05)$.

PCR assay. To demonstrate integration and transcription of fulllength TALE DNA in lentiviral infections of HeLa cells, the TALE repeat moiety was amplified from genomic DNA and cDNA, respectively, with Herculase II Fusion DNA polymerase (TALE repeat primers: forward, 5'-CCAGTTGCTGAAGATCGCGAAGC-3'; reverse, 5'-TGCCACTCGATGTGATGTCCTC-3'). The woodchuck hepatitis virus post-transcriptional regulatory element (WPRE) present in lentiviral constructs was used as a control (forward, 5'-AGCGTCGACAATCAACCTCT-3'; reverse, 5'-GGCATTAAAGCAGCGTATCC-3'). PCR products were purified using the QIAquick PCR Purification Kit (Qiagen $\mathrm{GmbH}$ ), and $100 \mathrm{ng}$ of each product was run on a $0.8 \%$ agarose gel.

HeL a cell lentivirus transduction. HeLa cells were seeded at a density of $1 \times 10^{6}$ cells per $10 \mathrm{~cm}^{3}$ in DMEM culture medium supplemented with $10 \%$ FBS and penicillin/streptomycin. Twenty-four hours after seeding, cells were treated with $1 \times 10^{7}$ titration units of lentivirus. Polybrene was added at a concentration of $8 \mathrm{ng} / \mu \mathrm{l}$ to enhance viral infection. Cells were harvested 4 days after infection for either DNA/ RNA extraction or preparation of protein lysate as described.

Western blotting. Following transduction, cells were sedimented and homogenized in radioimmunoprecipitation assay buffer. Cell lysates were sonicated, and sedimented to remove cellular debris, and protein concentration was measured by the Millipore Direct Detect system. Fifty micrograms of protein lysate was denatured with DTT and separated by size on a NuPAGE $4 \%-12 \%$ Bis-Tris gel (Life Technologies). Samples were transferred onto a PVDF membrane (Novex; Life Technologies) and blocked with PBST 5\% nonfat dry milk. Membranes were incubated with anti-FLAG antibody (F1804-200UG monoclonal ANTI-FLAG M2 antibody; Sigma Life Sciences) and anti$\beta$-actin antibody ( $\beta$-actin antibody 4967; Cell Signaling Technology Inc.), and then with HRP-conjugated secondary antibodies (sc-2030 goat anti-rabbit IgG-HRP and sc-2005 goat anti-mouse IgG-HRP; Santa Cruz Biotechnology). Blots were developed using ECL Prime Western Blotting Detection Reagent (GE Healthcare).

Primary human fibroblast transduction. Primary human foreskin fibroblasts purchased from ATCC, CCD-1112Sk (ATCC CRL-2429), were seeded at a density of $1 \times 10^{6}$ cells per $10 \mathrm{~cm}^{3}$ in Iscove's modified Dulbecco's medium supplemented with $10 \%$ FBS and penicillin/ streptomycin. After 24 hours, cells were infected with $1 \times 10^{7}$ titration units of lentivirus in complete medium with polybrene at a concentration of $8 \mathrm{ng} / \mu \mathrm{l}$ and harvested 4 days later using the Qiagen AllPrep DNA/RNA Mini Kit.

Primary human coronary artery smooth muscle cell transduction. Primary human coronary artery smooth muscle cells were purchased from Lonza (CC-2583) and plated in 6-well plates at a density of 75,000 cells per well in culture media prepared from the Lonza Clonetics SmGM-2 BulletKit (CC-3182). After 24 hours in culture, cells were infected with lentivirus as described for human fibroblasts.

$E d U$ incorporation. Primary human fibroblasts were plated in 8-well chamber slides and infected with either p16 jTALE-DNMT 
WT or mutant lentivirus as described above. After 72 hours in culture, cells were incubated with 5-ethynyl-2'-deoxyuridine (EdU) for 1 hour. EdU incorporation was visualized by immunofluorescence staining using the Click-iT Plus EdU Imaging Kit, Alexa Fluor 555 picolyl azide (Molecular Probes; Life Technologies). Percentage EdU incorporation was calculated as the number of EdU-positive cells divided by the total number of cells $(n=7)$. Differences in percentage EdU incorporation were compared in a 2 -tailed $t$ test $(P<0.05)$.

p16 re-expression. For p16 re-expression experiments, a lentiviral vector incorporating the human $p 16$ cDNA clone under a CMV promoter was purchased from OriGene Technologies Inc. (catalog RC220937L1). Lentivirus was prepared by The Wistar Institute Protein Expression Core. In separate EdU incorporation experiments, CMV-p16 lentivirus was added in combination with either p16 jTALEDNMT WT or mutant lentivirus. Titers for CMV-p16 lentivirus were reduced to approximately $1 \times 10^{6}$ titration units per 1 million cells. Percentage EdU incorporation was calculated as the number of EdUpositive cells divided by the total number of cells $(n=4)$. Differences in percentage EdU incorporation were compared in a 2-tailed $t$ test.

Population doubling time assay. Human fibroblasts were infected as described above, except that a lower seeding density of $4 \times 10^{5}$ cells per $10-\mathrm{cm}^{3}$ plate was used to prevent contact growth inhibition during the 4-day infection period. Cells were counted before plating and at the end of the experiment. Population doubling time (DT) was calcu- lated as DT $=T \ln 2 / \ln (\mathrm{Xf} / \mathrm{Xi})$, where $T$ is incubation time (days), $\mathrm{Xi}$ is initial cell number, and $\mathrm{Xf}$ is final cell number.

Statistics. Data are displayed as mean \pm SEM, and at least 3 replicates were conducted for each experiment. Data were assessed by 1-way ANOVA with Tukey's correction for multiple comparisons or by 2 -tailed $t$ test, as appropriate. Significance was defined as $P<0.05$.

Study approval. All experiments were performed in accordance with guidelines set forth by the University of Pennsylvania. Study approval was not required since no animals were used and primary human tissues were obtained from commercially available sources.

\section{Acknowledgments}

We thank the University of Pennsylvania Diabetes Research Center for the use of the Functional Genomics Core (P30-DK019525) and Aryel Heller for technical assistance. This work was supported by NIH grants U01-DK089529, UC4-DK104119, and R01DK088383 to K.H. Kaestner, and training grant T32GM008076.

Address correspondence to: Klaus H. Kaestner, Department of Genetics and Institute for Diabetes, Obesity, and Metabolism, 3400 Civic Center Boulevard, Perelman School of Medicine, University of Pennsylvania, Philadelphia, Pennsylvania 191046145, USA. Phone: 215.898.8759; E-mail: kaestner@mail.med. upenn.edu.
1. Heerboth S, Lapinska K, Snyder N, Leary M, Rollinson S, Sarkar S. Use of epigenetic drugs in disease: an overview. Genet Epigenet. 2014;6:9-19.

2. Cacabelos R. Epigenomic networking in drug development: from pathogenic mechanisms to pharmacogenomics. Drug Dev Res. 2014;75(6):348-365.

3. Jones PA. Functions of DNA methylation: islands, start sites, gene bodies and beyond. Nat Rev Genet. 2012;13(7):484-492.

4. Bergman Y, Cedar H. DNA methylation dynamics in health and disease. Nat Struct Mol Biol. 2013;20(3):274-281.

5. Sheaffer KL, et al. DNA methylation is required for the control of stem cell differentiation in the small intestine. Genes Dev. 2014;28(6):652-664.

6. Siddique AN, et al. Targeted methylation and gene silencing of VEGF-A in human cells by using a designed Dnmt3a-Dnmt3L single-chain fusion protein with increased DNA methylation activity. J Mol Biol. 2013;425(3):479-491.

7. Bogdanove AJ, Voytas DF. TAL effectors: customizable proteins for DNA targeting. Science. 2011;333(6051):1843-1846.

8. Cermak T, et al. Efficient design and assembly of custom TALEN and other TAL effector-based constructs for DNA targeting. Nucleic Acids Res. 2011;39(12):e82.

9. Morbitzer R, Romer P, Boch J, Lahaye T. Regulation of selected genome loci using de novoengineered transcription activator-like effector
(TALE)-type transcription factors. Proc Natl Acad Sci U S A. 2010;107(50):21617-21622.

10. Cong L, Zhou R, Kuo Y, Cunniff M, Zhang F. Comprehensive interrogation of natural TALE DNA-binding modules and transcriptional repressor domains. Nat Commun. 2012;3:968.

11. Maeder ML, et al. Targeted DNA demethylation and activation of endogenous genes using programmable TALE-TET1 fusion proteins. Nat Biotechnol. 2013;31(12):1137-1142.

12. Mendenhall EM, et al. Locus-specific editing of histone modifications at endogenous enhancers. Nat Biotechnol. 2013;31(12):1133-1136.

13. Holkers M, et al. Differential integrity of TALE nuclease genes following adenoviral and lentiviral vector gene transfer into human cells. Nucleic Acids Res. 2013;41(5):e63.

14. Martin N, Beach D, Gil J. Ageing as developmental decay: insights from p16INK4a. Trends $\mathrm{Mol}$ Med. 2014;20(12):667-674.

15. Jeck WR, Siebold AP, Sharpless NE. Review: a meta-analysis of GWAS and age-associated diseases. Aging Cell. 2012;11(5):727-731.

16. Herman JG, et al. Inactivation of the CDKN2/ p16/MTS1 gene is frequently associated with aberrant DNA methylation in all common human cancers. Cancer Res. 1995;55(20):4525-4530.

17. Krishnamurthy J, et al. p16(INK4a) induces an age-dependent decline in islet regenerative potential. Nature. 2006;443(7110):453-457.
18. Sanjana NE, Cong L, Zhou Y, Cunniff MM, Feng G, Zhang F. A transcription activator-like effector toolbox for genome engineering. Nat Protoc. 2012;7(1):171-192.

19. Rivenbark AG, et al. Epigenetic reprogramming of cancer cells via targeted DNA methylation. Epigenetics. 2012;7(4):350-360.

20. Kent WJ, et al. The human genome browser at UCSC. Genome Res. 2002;12(6):996-1006.

21. Ivanova TA, et al. Up-regulation of expression and lack of 5' $\mathrm{CpG}$ island hypermethylation of p16 INK4a in HPV-positive cervical carcinomas. BMC Cancer. 2007;7:47.

22. McLaughlin-Drubin M, Park D, Munger K. Tumor suppressor p16INK4A is necessary for survival of cervical carcinoma cell lines. Proc Natl Acad Sci U S A . 2013;110(40):16175-16180.

23. Yang L, et al. Optimization of scarless human stem cell genome editing. Nucleic Acids Res. 2013;41(19):9049-9061.

24. Sander JD, Joung JK. CRISPR-cas systems for editing, regulating and targeting genomes. Nat Biotechnol. 2014;32(4):347-355.

25. Doyle EL, et al. TAL effector-nucleotide targeter (TALE-NT) 2.0: tools for TAL effector design and target prediction. Nucleic Acids Res. 2012;40(Web Server issue):W117-W122.

26. Chen P, Cokus SJ, Pellegrini M. BS Seeker: precise mapping for bisulfite sequencing. BMC Bioinformatics. 2010;11:203. 